\title{
Prospective natural history study of central nervous system hemangioblastomas in von Hippel-Lindau disease
}

\author{
Clinical article
}

\author{
Russell R. Lonser, M.D., ${ }^{1,2}$ John A. Butman, M.D., Ph.D., ${ }^{3}$ Kristin Huntoon, Ph.D., 1 \\ Ashok R. Asthagiri, M.D., ${ }^{1}$ Tianxia Wu, Ph.D., ${ }^{4}$ Kamran D. Bakhtian, M.S., ${ }^{1}$ \\ Emily Y. Chew, M.D., ${ }^{5}$ Zhengping Zhuang, M.D., Ph.D., ${ }^{1}$ W. Marston Linehan, M.D. ${ }^{6}$ \\ AND EdWARd H. OLdFIELd, M.D., ${ }^{1,7}$ \\ ${ }^{1}$ Surgical Neurology Branch and ${ }^{4}$ Office of the Clinical Director, National Institute of Neurological \\ Disorders and Stroke; ${ }^{3}$ Neuroradiology Section, Diagnostic Radiology, Clinical Center at the National \\ Institutes of Health; ${ }^{5}$ Division of Epidemiology and Clinical Applications, National Eye Institute; ${ }^{6}$ Urologic \\ Oncology Branch, Center for Cancer Research, National Institutes of Health, Bethesda, Maryland; \\ ${ }^{2}$ Department of Neurological Surgery, Ohio State University Wexner Medical Center, Columbus, Ohio; \\ and ${ }^{7}$ Department of Neurological Surgery, University of Virginia, Charlottesville, Virginia
}

Object. The tumors most frequently associated with von Hippel-Lindau (VHL) disease are hemangioblastomas. While they are associated with significant neurological impairment and mortality, their natural history and optimal management have not been fully defined.

Methods. Patients with VHL were enrolled in a prospective study designed to define the natural history of CNS hemangioblastomas. In the present analysis, serial imaging, laboratory, genetic, and clinical data were evaluated in those with at least 2 years of follow-up data.

Results. At study entrance 225 patients (111 males, 114 females) harbored 1921 CNS hemangioblastomas in the supratentorial compartment (21 tumors [1\%]), cerebellum (865 [45\%]), brainstem (129 [7\%]), spinal cord (689 [36\%]), cauda equina (212 [11\%]), and nerve roots (5 [0.3\%]; follow-up 15,819 hemangioblastoma-years). Increased tumor burden was associated with partial deletions in the VHL gene $(\mathrm{p}=0.005)$ and male sex $(\mathrm{p}=0.002)$. Hemangioblastoma development (median 0.3 new tumors/year) was associated with younger age $(\mathrm{p}<0.0001)$ and more tumors at study entrance $(\mathrm{p}<0.0001)$. While 1278 hemangioblastomas $(51 \%)$ did not grow, 1227 hemangioblastomas $(49 \%)$ grew in a saltatory $(886[72 \%])$, linear $(76[6 \%])$, or exponential $(264[22 \%])$ pattern. Faster tumor growth was associated with male sex $(\mathrm{p}=0.001)$, symptomatic tumors $(\mathrm{p}<0.0001)$, and tumors associated with cysts $(\mathrm{p}<0.0001)$. Location-dependent tumor size was the primary predictor of eventual symptom formation (159 symptomatic tumors [6.3\%]; area under the curve $>0.9$ ).

Conclusions. Central nervous system hemangioblastoma burden in VHL is associated with partial germline deletions and male sex. Unpredictable growth of hemangioblastomas compromises assessment of nonsurgical therapies. The judicious treatment of symptom-producing hemangioblastomas, while avoiding unnecessary treatment of asymptomatic tumors that may not progress, can provide clinical stability. Clinical trial registration no.: NCT00005902 (ClinicalTrials.gov).

(http://thejns.org/doi/abs/10.3171/2014.1.JNS131431)

KEY WORDS • central nervous system $\bullet$ hemangioblastoma $\bullet$ natural history $\bullet$
von Hippel-Lindau disease $\bullet \quad$ oncology

$\mathrm{G}$ ERMLINE mutations of the von Hippel-Lindau (VHL) disease gene on chromosome 3 underlie the pathogenesis of this neoplastic disorder. Transmitted in an autosomal dominant manner, VHL disease can result in the development of tumors and cysts

Abbreviations used in this paper: $\mathrm{AUC}=$ area under the ROC curve; KPS = Karnofsky Performance Scale; ROC = receiver operating characteristic; VHL = von Hippel-Lindau. of the viscera and nervous system. ${ }^{16}$ The most common neoplastic manifestations of VHL are CNS hemangioblastomas. ${ }^{7,22}$ Prior reports estimate that multiple hemangioblastomas will develop in 60\%-90\% of patients with VHL disease. ${ }^{5,6,10,15,17,20,21,23}$ While CNS hemangioblastomas are benign, they are associated with significant neu-

This article contains some figures that are displayed in color online but in black-and-white in the print edition. 
rological morbidity and mortality based on their location and multiplicity. ${ }^{4}$ Despite the frequency and devastating effects of CNS hemangioblastomas in VHL, their natural history and optimal management have not been defined.

To determine the natural history of CNS hemangioblastomas, to gain insight into factors affecting tumor development and/or progression, and to improve tumor management, patients with VHL disease and associated hemangioblastomas were prospectively evaluated using clinical, imaging, and genetic analyses. Specifically, analyses were performed to determine the biological properties of CNS hemangioblastomas, as well as to identify factors associated with tumor burden, new tumor development, and the production of symptoms requiring treatment.

\section{Methods}

\section{Patients}

This study was registered with the ClinicalTrials.gov database (http://clinicaltrials.gov), and its registration no. is NCT00005902. Patients with at least one hemangioblastoma visible on MRI provided written informed consent and were enrolled in an institutional review boardapproved protocol to determine the natural history of VHL-associated hemangioblastomas (accrual ceiling 250 participants). Patients were enrolled from November 2001 to October 2005. All patients had genetic and/or clinical confirmation of VHL. ${ }^{12,19}$

\section{Germline Genotype Analysis}

Analysis of $V H L$ mutation was performed on samples of peripheral blood from the study participant or at least one family member to determine germline genotype, as described previously. ${ }^{26}$

\section{Study Evaluation}

Clinical Evaluation. Patients were evaluated at 6-month intervals. At each visit, detailed neurological examinations were performed and Karnofsky Performance Scale (KPS) scores were determined. Abdominal imaging and patient records were used to document the presence of visceral lesions.

Imaging Evaluation. Craniospinal high-resolution (1mm slice thickness) FLAIR and T1- and T2-weighted MRI (with and without contrast) was performed at each clinical evaluation.

\section{Hemangioblastoma Characteristics}

To accurately assess tumor distribution, development, and growth over time, patients and/or tumors with less than 2 years of follow-up were excluded from analysis. Data analysis for patients and/or tumors treated with systemic chemotherapy, stereotactic radiosurgery, or craniospinal radiation was terminated on initiation of those therapies. ${ }^{2,25}$

Postcontrast T1-weighted, spoiled gradient recalled, and T2-weighted MRI sequences were acquired at clinic visits and analyzed. Hemangioblastoma and associated cyst (if present) volumes were calculated using a modi- fied ellipsoid formula at each visit. ${ }^{14}$ Peritumoral cysts were assessed using T2-weighted (hyperintensity) MRI. Intratumoral cysts were evaluated using T1-weighted (hypointensity within enhancing tumor) postcontrast MRI. Volume assessment values were initially assessed serially by a single observer and confirmed by at least one other observer. Patterns of tumor growth were classified as saltatory (exhibiting periods of growth and growth quiescence), linear, or exponential. Tumors that did not progress in size were classified as stable.

\section{Statistical Analysis}

Descriptive statistics were used to summarize patient characteristics and hemangioblastoma features. To examine the effect of factors, such as age and sex, on tumor growth rate, a linear mixed model (MIXED procedure, SAS Institute Inc.) with the patient as random effect was used since most patients had more than 1 tumor. All tumors in the study had at least 2 years of follow-up and 4 data points.

Tumor growth pattern was determined with mathematical characterization. "No growth" was defined as the difference in tumor size $\leq 7.5 \mathrm{~mm}^{3} /$ year between baseline and the last visit divided by the years of follow-up. "Saltatory growth" was defined as the total number of days at zero-growing intervals divided by the total number of days of follow-up for the tumor ( $\geq 25 \%$ ), where the zerogrowing interval was defined as the interval with 0 difference between tumor size at adjacent time points. A tumor was defined as having "linear growth" if the $\mathrm{R}^{2}\left(\mathrm{R}_{1}{ }^{2}\right)$ from the regression of tumor size (dependent variable) on the visit date (independent variable) was $\geq 0.85$ and greater than or equal to the $\mathrm{R}^{2}\left(\mathrm{R}_{2}{ }^{2}\right)$ from the regression of log-transformed tumor size (dependent variable) on the visit date. A tumor was defined as having "exponential growth" if $\mathrm{R}_{2}^{2}$ was $\geq 0.85$ and $>\mathrm{R}_{1}{ }^{2}$.

A general linear model (GLM procedure, SAS Institute Inc.) was applied to examine the association of patient total number of tumors with age (categorized as $12-20,21-30,31-40,41-50$, or $>50$ years), sex, years of follow-up, and mutation genotype. A general linear model was also applied to determine the association of newly developed tumor per year with age (categorized), sex, number of tumors at study entrance, and mutation genotype. Pedigree effect was examined using a mixed model (MIXED procedure). Since most patients had more than 1 tumor and the tumors from the same patient were not independent, a general linear mixed model with subject as the random effect (MIXED procedure) was used to assess the association of tumor growth rate with age, sex, tumor location, cyst-linked tumor type (4 levels, including linked to intratumoral cyst, linked to peritumoral cyst, linked to both cysts, and linked to none), and tumor symptom type (that is, asymptomatic or symptomatic tumor). A p value of 0.1 was used as a model selection criterion for the above association analysis.

To examine the effect of factors on a patient's total number of tumors and number of newly developed tumors per year, a general linear model was performed since pedigree effect was not significant. Receiver operating characteristic (ROC) curve analysis using a general- 
ized mixed model (GLIMMIX procedure) and a logistic regression model (LOGISTIC procedure, both SAS Institute Inc.) was applied to evaluate tumor volume for the prediction of symptomatic tumors. Discrimination was assessed using the area under the ROC curve (AUC). Survival analysis was performed using growth as the event of interest, defined as the difference in tumor volume $>7.5$ $\mathrm{mm}^{3}$ between the last and first evaluation, and not growing as censored. The timing of events was determined using the time the tumor was detectable on imaging (12 $\mathrm{mm}^{3}$ ) to a time in which growth was $>7.5 \mathrm{~mm}^{3}$.

Quantitative outcome measures were logarithmically transformed because their distributions had a long right tail. For any fixed factor with more than 2 levels in which significance was found, Tukey-Kramer multiple pairwise comparisons were performed. A p value of 0.01 was used as the significance level to adjust for multiple tests, and a $p$ value of 0.1 was used as the covariate selection. For statistical analyses, SAS software version 9.2 was used (SAS Institute Inc.).

\section{Results}

\section{Patient Characteristics}

Two hundred fifty patients with VHL disease (119 males, 131 females) were enrolled in the study. The mean age at study entrance was $38.5 \pm 12.3$ years (range 12.3-66.1 years). Two hundred twenty-five patients (90\%) had tumors as well as more than 2 years of follow-up and were used for analysis (Table 1). The mean followup was $6.9 \pm 1.6$ years (range 2.1-9.0 years). During the study, 16 patients died (mean age $41.9 \pm 10.9$ years, range 17.7-56.3 years) from renal cell carcinoma (2 patients), CNS hemangioblastoma progression (4 patients), stroke (1 patient), pneumonia (1 patient), and unknown causes (8 patients). Twenty-five patients $(10 \%)$ had less than 2

TABLE 1: Lesions associated with VHL disease in 225 patients at study entrance

\begin{tabular}{lc}
\hline \multicolumn{1}{c}{ Lesion } & No. of Patients (\%) \\
\hline nervous system & \\
craniospinal hemangioblastoma & \\
supratentorial & $23(10.2)$ \\
cerebellum & $197(87.6)$ \\
brainstem & $94(41.8)$ \\
spinal cord & $193(85.8)$ \\
cauda equina & $139(61.8)$ \\
nerve root & $5(2.2)$ \\
retinal hemangioblastoma & $118(52.4)$ \\
endolymphatic sac tumor & $29(12.9)$ \\
visceral lesions & \\
renal cell carcinoma & $131(58.2)$ \\
renal cyst & $144(64.0)$ \\
pancreatic neuroendocrine tumor & $75(33.3)$ \\
pancreatic cyst & $130(57.8)$ \\
pheochromocytoma & $47(20.9)$ \\
reproductive adnexal organ cystadenoma & $71(31.6)$ \\
\hline
\end{tabular}

years of evaluation as a result of death (7 patients), study withdrawal (5 patients), or loss to follow-up (13 patients).

\section{Hemangioblastoma Features}

Hemangioblastoma Burden. At study entrance, the 225 patients harbored 1921 craniospinal hemangioblastomas (mean $8.5 \pm 7.0$ tumors/patient, median 7 tumors/ patient, range 1-33 tumors/patient) located in the supratentorial compartment (21 tumors [1\%]), brainstem (129 [7\%]), cerebellum (865 [45\%]), spinal cord (689 [36\%]), cauda equina (212 [11\%]), and nerve roots (5 [0.3\%]; Table 1). The mean total tumor volume per patient upon study entrance was $562.8 \pm 1306.6 \mathrm{~mm}^{3}$ (median $182.5 \mathrm{~mm}^{3}$, range $12-11,919.9 \mathrm{~mm}^{3}$ ).

At the last evaluation, patients harbored 2336 craniospinal hemangioblastomas (mean $10.4 \pm 7.8$ tumors/ patient, median 8 tumors/patient). Hemangioblastomas were found in the supratentorial compartment (21 tumors [1\%]), brainstem (132 tumors [6\%]), cerebellum (1049 tumors [45\%]), spinal cord (867 tumors [37\%]), cauda equina (261 tumors [11\%]), and nerve roots (6 tumors [0.3\%]). The mean total craniospinal hemangioblastoma volume per patient was $1618.4 \pm 3734.7 \mathrm{~mm}^{3}$ (median 369.1 $\mathrm{mm}^{3}$, range $0-28,304.5 \mathrm{~mm}^{3}$ ). Total tumor follow-up was 15,819 hemangioblastoma-years.

New Hemangioblastoma Development. One hundred sixty-two patients (72\%) exhibited new CNS hemangioblastomas during the study. Consistent with the overall anatomical distribution of tumors, new hemangioblastomas developed in the supratentorial compartment (4 [0.7\%]), cerebellum (291 [50\%]), brainstem (16 [3\%]), spinal cord (217 [37\%]), cauda equina (54 [9\%]), or nerve roots (2 [0.3\%]). Over the study duration, a mean of $0.4 \pm 0.4$ new tumors/year/patient developed (median 0.3 tumors).

Hemangioblastoma Growth. Twelve hundred seventy-eight hemangioblastomas (51\% of tumors evaluated) remained stable and 1227 (49\%) grew during the study. Progression was observed in $25 \%$ of tumors followed up for 1.5-5.0 years and in 50\% of tumors followed up for 5.6-8.9 years, depending on tumor location (Fig. 1). Patterns of growth varied and were categorized as saltatory (886 tumors [72\%]), linear (76 [6\%]), or exponential (264 [22\%]; Fig. 2). The median growth rate was $3.7 \mathrm{~mm}^{3} /$ year (range $0.8-2331.9 \mathrm{~mm}^{3} / \mathrm{year}$ ) for tumors growing in a saltatory pattern, $23.8 \mathrm{~mm}^{3} /$ year (range $0.8-1245.4 \mathrm{~mm}^{3} /$ year) in a linear pattern, and $79.2 \mathrm{~mm}^{3} /$ year (range 1.8 $6216.6 \mathrm{~mm}^{3} /$ year $)$ in an exponential pattern $(\mathrm{p}<0.0001$ for all pairwise comparisons).

While the various patterns of growth were represented among the different anatomical regions, hemangioblastomas grew at significantly different rates depending on their anatomical region. Hemangioblastomas located in the brainstem (median $3.1 \mathrm{~mm}^{3} /$ year) and cerebellum (median $1.2 \mathrm{~mm}^{3} /$ year) grew significantly faster than those arising in the spinal cord (median $0.3 \mathrm{~mm}^{3} / \mathrm{per}$ year) or cauda equina (median $0 \mathrm{~mm}^{3} /$ year; $\mathrm{p}<0.0001$ ).

Hemangioblastoma-Associated Cysts. During the study, 295 hemangioblastomas developed cysts. Two hun- 


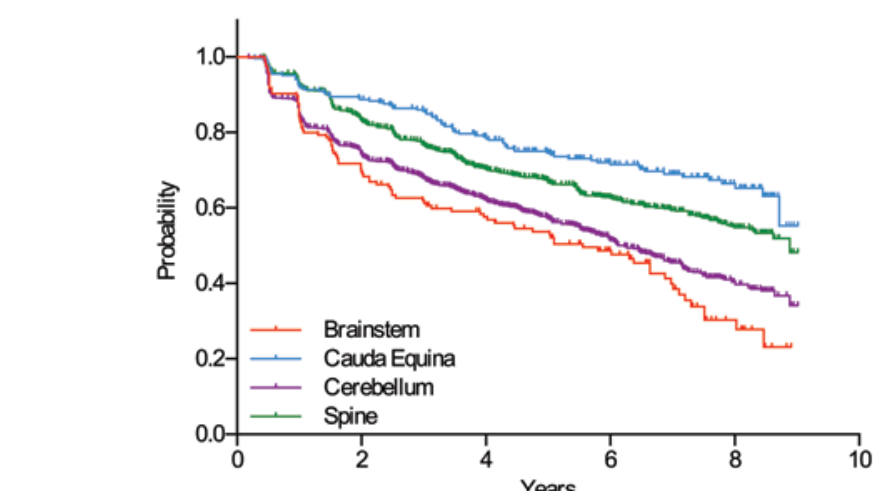

No. at Risk Brainstem Cauda Equina Cerebellum Spine

$\begin{array}{rr}145 & 10 \\ 266 & 236 \\ 1156 & 860 \\ 906 & 75\end{array}$

101
236
860
755

$\begin{array}{rr}76 & 46 \\ 190 & 129 \\ 608 & 334 \\ 551 & 347\end{array}$

12
55
116

12
55
116

No. at Risk

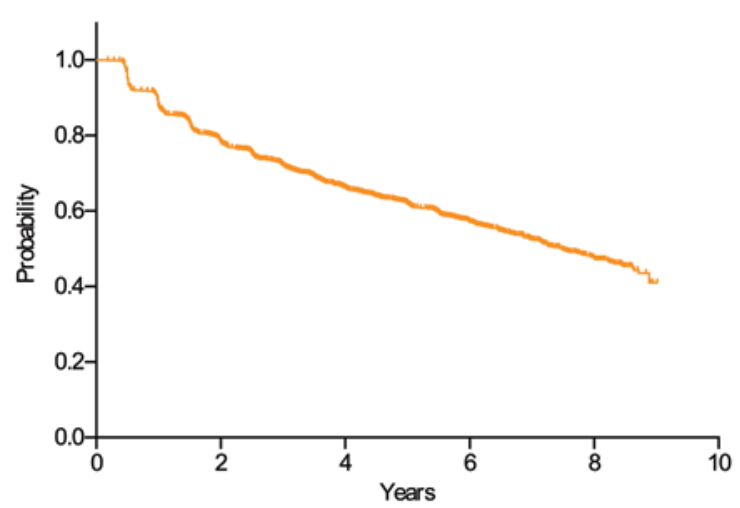

FIG. 1. Kaplan-Meier analysis of hemangioblastoma progression over an 8-year period in patients with VHL disease. Left: Fifty percent of (nongrowing) hemangioblastomas located in the brainstem progressed in 5.6 years. Twenty-five percent of hemangioblastomas located in the cauda equina progressed in 5.0 years. Fifty percent of hemangioblastomas located in the cerebellum progressed in 6.2 years. Fifty percent of hemangioblastomas located in the spine progressed in 8.9 years. Right: Twenty-five percent of hemangioblastomas at all tumor locations progressed in 2.5 years and $50 \%$ in 7.5 years. No. at risk = tumors at risk for growing. The numbers of tumors at risk refer to the years on the $x$-axis under which they align.

dred forty-seven tumors (9.9\% of total 2505) developed peritumoral cysts, $37(1.5 \%)$ developed intratumoral cysts, and $11(0.4 \%)$ developed both types of cyst. Five cysts arose in the supratentorial compartment $(1.7 \%), 190$ in the cerebellum (64.4\%), 32 in the brainstem (10.8\%), 67 in the spinal cord $(22.7 \%)$, and 1 in a nerve root $(0.3 \%)$.

Tumors associated with cysts demonstrated increased lesion volume, growth rate, and production of symptoms. For instance, there were 100 symptomatic cerebellar tumors $(8.6 \%)$ among 1156 cerebellar hemangioblastomas, and 60 were associated with peritumoral cysts, as compared with $89(8.4 \%)$ of the 1056 cerebellar hemangioblastomas without symptoms. The ratio of the median growth rate of peritumoral cysts to the tumor growth rate for symptomatic cerebellar hemangioblastomas was 22:1, and the ratio of the median volume of the peritumoral cyst compared with tumor was 10:1.

Hemangioblastoma Symptom Association. While 2346 hemangioblastomas were asymptomatic (93.6\%) at the final evaluation, 159 hemangioblastomas (6.3\%) in 75 patients produced symptoms and required treatment. Neurological symptoms and signs were caused by mass effect related to hemangioblastoma and/or cyst size in their specific anatomical location (Table 2). Fourteen of the hemangioblastomas (8.8\%) that became symptomatic were not present on imaging at study entrance.

\section{Clinical Progression}

The KPS score remained unchanged in 136 patients
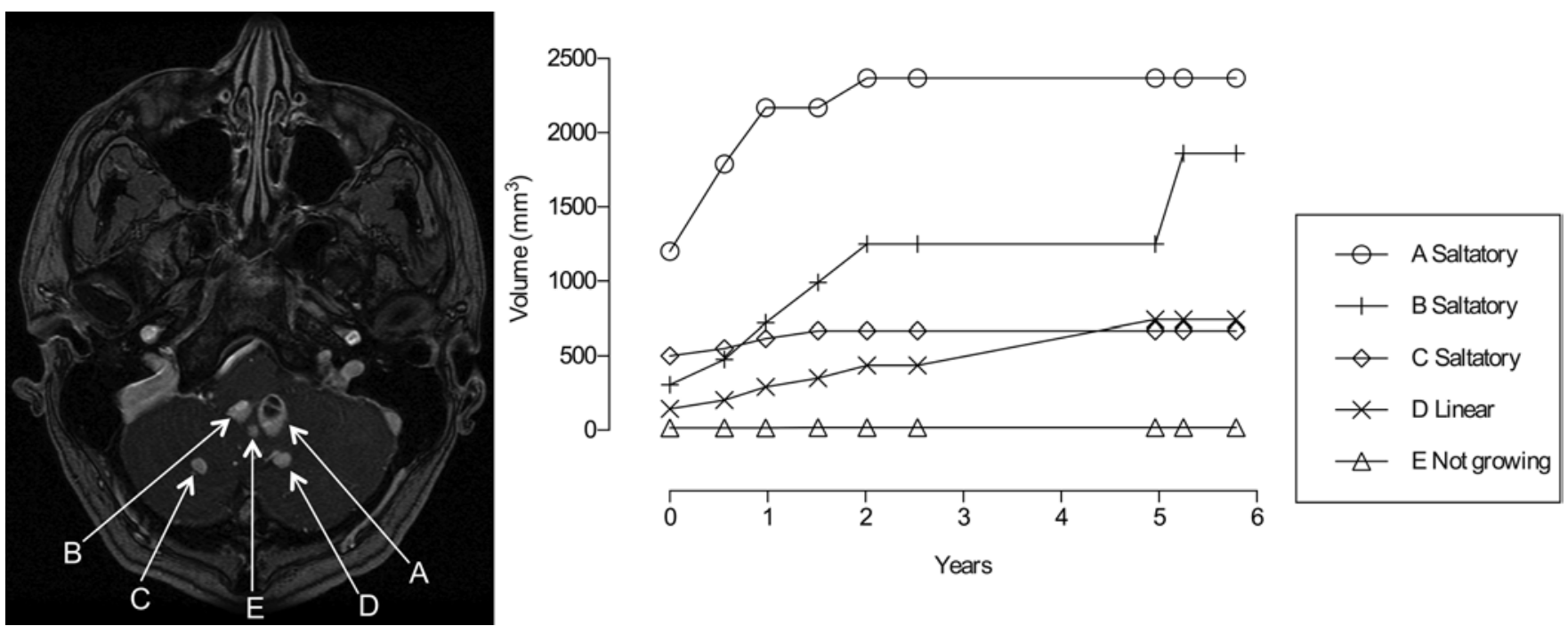

FIG. 2. Characteristic patterns of growth associated with CNS hemangioblastomas in patients with VHL disease. Left: Axial contrast-enhanced MR image of the cerebellum in a patient showing 5 tumors (A-E) that demonstrated 3 growth patterns. Right: Tumor growth patterns, including saltatory $(A-C)$, linear $(D)$, and stable $(E)$. 
TABLE 2: Symptoms based on anatomical location of 159 tumors*

\begin{tabular}{lc}
\hline \multicolumn{1}{c}{ Lesion Location } & $\begin{array}{c}\text { Frequency of Symptomatic } \\
\text { Lesions }(\%)\end{array}$ \\
\hline brainstem & \\
headache & $10(83.3)$ \\
singultus & $8(66.7)$ \\
nausea/vomiting & $6(50.0)$ \\
dysphagia & $5(41.7)$ \\
cough & $3(25.0)$ \\
paresthesia & $3(25.0)$ \\
cauda equina & \\
urinary/bowel abnormalities & $5(100.0)$ \\
pain & $5(100.0)$ \\
paresthesia & $4(80.0)$ \\
headache & $2(40.0)$ \\
cerebellum & \\
headache & $77(77.0)$ \\
gait ataxia & $57(57.0)$ \\
nausea/vomiting & $19(19.0)$ \\
vertigo & $18(18.0)$ \\
speech difficulties & $15(15.0)$ \\
dysmetria & $11(11.0)$ \\
nerve root & \\
cruciate paralysis & $1(3.3)$ \\
paresthesia & $28(73.7)$ \\
pain & $24(63.2)$ \\
gait ataxia & $13(34.2)$ \\
dysesthesias & $9(23.7)$ \\
urinary/bowel abnormalities & $7(18.4)$ \\
weaknententorial & \\
\hline
\end{tabular}

* The symptoms listed here are the major symptoms recorded in patients who underwent resection of symptomatic tumors. Patients who underwent resections at outside institutions are not included in this analysis because of limited records.

(60\%), improved in 32 (14\%; all associated with resection of symptom-producing hemangioblastomas), and worsened in $57(25 \%)$. Declines in KPS scores during the study were a result of disease progression (45 patients [20\% of all patients]), postoperative morbidity (10 patients [4\%]), or unknown reasons (2 patients [1\%]).

\section{Analysis of Factors Associated With Tumor Behavior}

Tumor Burden. Factors associated with an increased tumor burden included male sex $(p=0.002$; mean 11.5 tumors/male patient vs 9.2 tumors/female patient; median 9 vs 7 , respectively), longer follow-up $(\mathrm{p}=0.007)$, and genotype $(\mathrm{p}=0.002$; Table 3$)$. Specifically, patients harboring partial germline deletions (60 patients [27\%]) had more tumors than patients with missense mutations (86 patients [38\%]; $\mathrm{p}=0.005$ ).

New Tumor Development. Sex and genotype had no effect on the development of new tumors (Table 3). Patients with a greater tumor burden at study entrance demonstrated more tumors over the course of the study ( $p$ $<0.0001)$. Age had a significant ( $\mathrm{p}<0.0001)$ effect on tumor development. Patients 12-20 years of age developed more tumors per year than older groups.

Tumor Growth. Tumors grew faster in males than in females $(p=0.001$; Table 3$)$. The effects of tumor growth rate on the presence and type of symptoms varied with location ( $p<0.0001)$. For each anatomical compartment, the symptom-producing tumors grew faster than the asymptomatic ones $(p<0.0001)$. Symptom-producing tumors in the spine grew significantly slower than those in the cerebellum ( $\mathrm{p}=0.0007)$. Asymptomatic tumors in the spine and cauda equina (no significant difference between these 2 regions, $\mathrm{p}=1.0)$ grew significantly $(\mathrm{p}<0.0001)$ slower than those in the brainstem and cerebellum (no significant difference between these 2 regions, $p=0.7$ ). For each anatomical compartment, tumors associated with a cystic component grew substantially faster than tumors not associated with cysts $(\mathrm{p}<0.0001)$. For the cerebellar compartment, symptomatic tumors associated with intratumoral cysts grew faster than those associated with peritumoral cysts $(\mathrm{p}<0.0001)$.

Cyst Growth. All types of cerebellar cysts (peritumoral, intratumoral, or combination of the two) grew at greater rates in younger patients $(p=0.01$; Table 3$)$. Peritumoral cysts grew faster in symptomatic $(p<0.0001)$ than in asymptomatic hemangioblastomas.

Factors That Predict Symptom Formation. Receiver operating characteristic analysis demonstrated that thresholds of location-dependent tumor size predict symptom formation (AUC of 0.99, 0.94, and 0.97 for brainstem, cerebellum, and spine, respectively). When specificity was set to at least $90 \%$, cerebellar hemangioblastomas reaching a diameter of $5.6 \mathrm{~mm}$, brainstem hemangioblastomas reaching a diameter of $4.0 \mathrm{~mm}$, and spinal cord hemangioblastomas reaching a diameter of $4.5 \mathrm{~mm}$ would be diagnosed as symptomatic with a sensitivity of $78 \%$ (specificity 96\%), 53\% (specificity 92\%), and 78\% (specificity 95\%), respectively.

\section{Discussion}

\section{Previous Reports}

Clinical studies have been used to develop successful paradigms for the treatment of VHL-associated visceral tumors (renal cell carcinoma, pancreatic neuroendocrine tumors, and pheochromocytomas) $11,27,28$ and endolymphatic sac tumors. ${ }^{3,18}$ Previous reports defining the natural history of VHL-associated hemangioblastomas have been inconclusive because of their retrospective analyses, inadequate numbers of patients, and limited follow-up, ${ }^{1,17,24,29}$ Given these limitations, the natural history of CNS hemangioblastomas, the timing for removing VHL-associated CNS 
TABLE 3: Analysis of factors associated with tumor behavior*

\begin{tabular}{|c|c|}
\hline Outcome Measure \& Factor & $p$ Value \\
\hline \multicolumn{2}{|l|}{ total tumor burden (no. of tumors; 225 patients) } \\
\hline age & NS \\
\hline yrs of follow-up & 0.0070 \\
\hline $\operatorname{sex}$ & 0.002 \\
\hline mutation† & 0.0021 \\
\hline partial deletion & reference \\
\hline deletion & 0.0667 \\
\hline frameshift & 0.0450 \\
\hline missense & 0.0046 \\
\hline nonsense & 0.2508 \\
\hline \multicolumn{2}{|l|}{ new tumor development (no. of tumors; 225 patients) } \\
\hline $\operatorname{sex}$ & NS \\
\hline mutation† & NS \\
\hline no. of tumors at study entrance & $<0.0001$ \\
\hline age in yrs & $<0.0001$ \\
\hline $12-20$ & reference \\
\hline $21-30$ & 0.0224 \\
\hline $31-40$ & 0.0005 \\
\hline $41-50$ & $<0.0001$ \\
\hline$>50$ & $<0.0001$ \\
\hline \multicolumn{2}{|l|}{ tumor growth rate (mm³/yr; 2505 tumors) } \\
\hline age & NS \\
\hline mutation† & NS \\
\hline $\operatorname{sex}$ & 0.0014 \\
\hline tumor location & $<0.0001$ \\
\hline tumor symptom type & $<0.0001$ \\
\hline tumor location × tumor symptom type & 0.0001 \\
\hline \multicolumn{2}{|l|}{ cerebellum tumor growth rate (mm³/yr; 1156 tumors) } \\
\hline age & NS \\
\hline mutation† & NS \\
\hline sex & NS \\
\hline cyst-linked tumor type & $<0.0001$ \\
\hline tumor symptom type & $<0.0001$ \\
\hline cyst-linked tumor type $\times$ tumor symptom type & $<0.0001$ \\
\hline \multicolumn{2}{|l|}{ cerebellum cyst growth rate (mm³/yr; 175 cysts) } \\
\hline sex & NS \\
\hline mutation† & NS \\
\hline age (continuous) & 0.0079 \\
\hline tumor symptom type & $<0.0001$ \\
\hline cyst type & NS \\
\hline tumor symptom type $\times$ cyst type & 0.0342 \\
\hline \multicolumn{2}{|c|}{$\begin{array}{l}\text { * Linear or linear mixed models were performed to evaluate the as- } \\
\text { sociation of factors with outcome measures. Age, sex, and mutation } \\
\text { would be dropped from the model if they were not significant based on } \\
\text { a significance level of } 0.1 \text {. NS }=\text { not significant. } \\
\dagger \text { Genotype was available for } 197 \text { of the } 225 \text { patients. }\end{array}$} \\
\hline
\end{tabular}

hemangioblastomas, and the role of radiosurgery and/or chemotherapy are unknown. To better understand features that can be used to guide management of CNS hemangioblastomas in VHL, we performed a long-term, prospective natural history study in a large cohort of patients.

\section{Biological and Clinical Implications}

Tumor Burden and New Tumor Development. Male sex was associated with increased CNS hemangioblastoma burden and growth rate, which may indicate that male hormonal influences affect the development and growth of CNS hemangioblastomas. This effect of sex on tumor development is consistent with previous findings that pregnancy did not impact hemangioblastoma development or progression in VHL patients of childbearing years. ${ }^{34}$

New hemangioblastomas developed in most patients $(72 \%)$. An increased rate of tumor development was associated with tumor burden at study entrance and younger age. These findings suggest that patients with more tumors at any point in time may be predisposed to additional tumors over a lifetime. Moreover, tumors developed in younger patients at a faster rate than in older patients. This finding indicates that CNS hemangioblastoma development in VHL may be affected by biological features related to developmental processes, hormonal factors, other systemic factors, and/or proteasomal processing.

Patients with partial germline deletions had an increased tumor burden as compared with the patients with missense mutations. Recent observations indicate that protein produced by missense germline alterations in neoplasia and metabolic syndromes, including VHL, ${ }^{33}$ can maintain intrinsic function. ${ }^{13,32}$ Further, the severity of disease burden and tumor development in patients with familial neoplasia syndromes may be the result of the rapid degradation of functional mutant protein via proteasome control pathways ${ }^{32}$ and/or an age-related decline in proteasome function of mutated tumor suppressor protein. ${ }^{9}$ These findings suggest that residual VHL protein (tumor suppressor) function associated with a germline missense mutation may underlie reduced disease severity.

Growth of Tumors. Consistent with prior retrospective studies, ${ }^{1,29}$ variable patterns of tumor growth were observed. While approximately half $(51 \%)$ of all CNS hemangioblastomas remained stable in size during longterm follow-up, $49 \%$ grew. The most common pattern of growth was saltatory ( $72 \%$ of growing tumors), followed by exponential (22\%) and linear (6\%). Because most tumors remain stable or grow in a saltatory pattern (characterized by prolonged periods, often years, of quiescence), extended periods of follow-up (probably 5 or more years) are necessary to accurately assess the efficacy of nonsurgical therapies, such as chemotherapy and radiation therapy, and tumor stability. Previously, we analyzed CNS hemangioblastomas treated with stereotactic radiosurgery in a cohort of patients with VHL (40 hemangioblastomas in 20 patients). ${ }^{2}$ At nearly 6 years after stereotactic radiosurgery, no progression occurred in 33\% of tumors, similar to the natural history of (untreated) CNS hemangioblastoma progression in the current study (25\%). 
Clinical Progression. Previous reports have indicated that patients with VHL have a shortened lifespan because of complications related to renal cell carcinoma and CNS hemangioblastomas..$^{30}$ Similarly, our study indicates that $25 \%$ of mortality was the result of VHL-associated tumor progression. Specifically, progression of CNS hemangioblastomas was the leading cause of VHL-associated mortality, followed by renal cell carcinoma-related disease. These findings differ from those in a number of previous reports that describe renal cell carcinoma as the leading cause of VHL-associated mortality ${ }^{4,17}$ and may reflect the universal occurrence of VHL-associated hemangioblastomas in the current study population.

The mainstay of therapy for sporadic and VHL-associated CNS hemangioblastomas is resection. Surgery can be safely performed in most patients. Better patient function in the current study was the result of resection of symptomatic CNS hemangioblastomas. These findings are similar to those in previous studies demonstrating symptom stability or improvement in most patients after resection of hemangioblastomas. ${ }^{8,31}$ Together, these results underscore the lasting clinical stability provided in most patients by the judicious treatment of symptom-producing hemangioblastomas and the avoidance of unnecessary therapies for asymptomatic tumors that may not progress.

\section{Conclusions}

Consistent with prior retrospective studies, ${ }^{1,29}$ signs and symptoms of hemangioblastomas are dictated by tumor location, associated edema, and cyst formation and propagation (Table 2) ${ }^{8}$ However, absolute size of the lesion did not dictate the symptoms for tumors in all locations, and neither did the rate of growth. Although a threshold size was used as the primary factor in the ROC modeling to predict eventual symptom formation, the likely time to symptom formation for individual lesions remains undefined because of the saltatory growth pattern exhibited by many tumors. These findings support the onset of symptoms associated with a particular CNS hemangioblastoma as the indication for surgery.

\section{Disclosure}

This research was supported by the Intramural Research Programs of the National Institute of Neurological Disorders and Stroke, the Clinical Center, National Eye Institute and the National Cancer Institute at the National Institutes of Health. The authors report no conflict of interest concerning the materials or methods used in this study or the findings specified in this paper.

Author contributions to the study and manuscript preparation include the following. Conception and design: Lonser, Oldfield. Acquisition of data: Lonser, Butman, Huntoon, Asthagiri, Bakhtian, Chew, Zhuang, Linehan, Oldfield. Analysis and interpretation of data: Lonser, Butman, Huntoon, Bakhtian, Chew, Zhuang, Oldfield. Drafting the article: Lonser, Huntoon, Bakhtian, Zhuang, Oldfield. Critically revising the article: all authors. Reviewed submitted version of manuscript: all authors. Approved the final version of the manuscript on behalf of all authors: Lonser. Statistical analysis: Huntoon, Wu. Administrative/technical/material support: Lonser, Bakhtian. Study supervision: Lonser, Oldfield.

\section{References}

1. Ammerman JM, Lonser RR, Dambrosia J, Butman JA, Old- field EH: Long-term natural history of hemangioblastomas in patients with von Hippel-Lindau disease: implications for treatment. J Neurosurg 105:248-255, 2006

2. Asthagiri AR, Mehta GU, Zach L, Li X, Butman JA, Camphausen KA, et al: Prospective evaluation of radiosurgery for hemangioblastomas in von Hippel-Lindau disease. Neuro Oncol 12:80-86, 2010

3. Butman JA, Kim HJ, Baggenstos M, Ammerman JM, Dambrosia J, Patsalides A, et al: Mechanisms of morbid hearing loss associated with tumors of the endolymphatic sac in von Hippel-Lindau disease. JAMA 298:41-48, 2007

4. Butman JA, Linehan WM, Lonser RR: Neurologic manifestations of von Hippel-Lindau disease. JAMA 300:1334-1342, 2008

5. Cockman ME, Masson N, Mole DR, Jaakkola P, Chang GW, Clifford SC, et al: Hypoxia inducible factor-alpha binding and ubiquitylation by the von Hippel-Lindau tumor suppressor protein. J Biol Chem 275:25733-25741, 2000

6. Filling-Katz MR, Choyke PL, Oldfield E, Charnas L, Patronas NJ, Glenn GM, et al: Central nervous system involvement in Von Hippel-Lindau disease. Neurology 41:41-46, 1991

7. Gläsker S: Central nervous system manifestations in VHL: genetics, pathology and clinical phenotypic features. Fam Cancer 4:37-42, 2005

8. Jagannathan J, Lonser RR, Smith R, DeVroom HL, Oldfield EH: Surgical management of cerebellar hemangioblastomas in patients with von Hippel-Lindau disease. J Neurosurg 108: 210-222, 2008

9. Kourtis N, Tavernarakis N: Cellular stress response pathways and ageing: intricate molecular relationships. EMBO J 30:2520-2531, 2011

10. Lamiell JM, Salazar FG, Hsia YE: von Hippel-Lindau disease affecting 43 members of a single kindred. Medicine (Baltimore) 68:1-29, 1989

11. Libutti SK, Choyke PL, Bartlett DL, Vargas H, Walther M, Lubensky I, et al: Pancreatic neuroendocrine tumors associated with von Hippel Lindau disease: diagnostic and management recommendations. Surgery 124:1153-1159, 1998

12. Lonser RR, Glenn GM, Walther M, Chew EY, Libutti SK, Linehan WM, et al: von Hippel-Lindau disease. Lancet 361: 2059-2067, 2003

13. Lu J, Yang C, Chen M, Ye DY, Lonser RR, Brady RO, et al: Histone deacetylase inhibitors prevent the degradation and restore the activity of glucocerebrosidase in Gaucher disease. Proc Natl Acad Sci U S A 108:21200-21205, 2011

14. Lundin P, Pedersen F: Volume of pituitary macroadenomas: assessment by MRI. J Comput Assist Tomogr 16:519-528, 1992

15. Maddock IR, Moran A, Maher ER, Teare MD, Norman A, Payne SJ, et al: A genetic register for von Hippel-Lindau disease. J Med Genet 33:120-127, 1996

16. Maher ER, Iselius L, Yates JR, Littler M, Benjamin C, Harris R, et al: Von Hippel-Lindau disease: a genetic study. J Med Genet 28:443-447, 1991

17. Maher ER, Yates JR, Harries R, Benjamin C, Harris R, Moore AT, et al: Clinical features and natural history of von HippelLindau disease. Q J Med 77:1151-1163, 1990

18. Manski TJ, Heffner DK, Glenn GM, Patronas NJ, Pikus AT, Katz D, et al: Endolymphatic sac tumors. A source of morbid hearing loss in von Hippel-Lindau disease. JAMA 277:14611466, 1997

19. Melmon KL, Rosen SW: Lindau's disease. Review of the literature and study of a large kindred. Am J Med 36:595-617, 1964

20. Neumann HP, Eggert HR, Scheremet R, Schumacher M, Mohadjer M, Wakhloo AK, et al: Central nervous system lesions in von Hippel-Lindau syndrome. J Neurol Neurosurg Psychiatry 55:898-901, 1992

21. Neumann HP, Lips CJ, Hsia YE, Zbar B: Von Hippel-Lindau syndrome. Brain Pathol 5:181-193, 1995 
22. Richard S, Campello C, Taillandier L, Parker F, Resche F: Haemangioblastoma of the central nervous system in von Hippel-Lindau disease. J Intern Med 243:547-553, 1998

23. Richard S, Chauveau D, Chrétien Y, Beigelman C, Denys A, Fendler JP, et al: Renal lesions and pheochromocytoma in von Hippel-Lindau disease. Adv Nephrol Necker Hosp 23:1-27, 1994

24. Richard S, David P, Marsot-Dupuch K, Giraud S, Béroud C, Resche F: Central nervous system hemangioblastomas, endolymphatic sac tumors, and von Hippel-Lindau disease. Neurosurg Rev 23:1-24, 2000

25. Simone CB II, Lonser RR, Ondos J, Oldfield EH, Camphausen K, Simone NL: Infratentorial craniospinal irradiation for von Hippel-Lindau: a retrospective study supporting a new treatment for patients with CNS hemangioblastomas. Neuro Oncol 13:1030-1036, 2011

26. Stolle C, Glenn G, Zbar B, Humphrey JS, Choyke P, Walther $\mathrm{M}$, et al: Improved detection of germline mutations in the von Hippel-Lindau disease tumor suppressor gene. Hum Mutat 12:417-423, 1998

27. Walther MM, Choyke PL, Glenn G, Lyne JC, Rayford W, Venzon D, et al: Renal cancer in families with hereditary renal cancer: prospective analysis of a tumor size threshold for renal parenchymal sparing surgery. J Urol 161:1475-1479, 1999

28. Walther MM, Reiter R, Keiser HR, Choyke PL, Venzon D, Hurley K, et al: Clinical and genetic characterization of pheochromocytoma in von Hippel-Lindau families: comparison with sporadic pheochromocytoma gives insight into natural history of pheochromocytoma. J Urol 162:659-664, 1999

29. Wanebo JE, Lonser RR, Glenn GM, Oldfield EH: The natural history of hemangioblastomas of the central nervous system in patients with von Hippel-Lindau disease. J Neurosurg 98: 82-94, 2003

30. Wilding A, Ingham SL, Lalloo F, Clancy T, Huson SM, Moran A, et al: Life expectancy in hereditary cancer predisposing diseases: an observational study. J Med Genet 49:264-269, 2012

31. Wind JJ, Bakhtian KD, Sweet JA, Mehta GU, Thawani JP, Asthagiri AR, et al: Long-term outcome after resection of brainstem hemangioblastomas in von Hippel-Lindau disease. Clinical article. J Neurosurg 114:1312-1318, 2011

32. Yang C, Asthagiri AR, Iyer RR, Lu J, Xu DS, Ksendzovsky A, et al: Missense mutations in the NF2 gene result in the quantitative loss of merlin protein and minimally affect protein intrinsic function. Proc Natl Acad Sci U S A 108:4980-4985, 2011

33. Yang C, Huntoon K, Ksendzovsky A, Zhuang Z, Lonser RR: Proteostasis modulators prolong missense VHL protein activity and halt tumor progression. Cell Rep 3:52-59, 2013

34. Ye DY, Bakhtian KD, Asthagiri AR, Lonser RR: Effect of pregnancy on hemangioblastoma development and progression in von Hippel-Lindau disease. Clinical article. J Neurosurg 117:818-824, 2012

Manuscript submitted July 4, 2013.

Accepted January 16, 2014.

Please include this information when citing this paper: published online February 28, 2014; DOI: 10.3171/2014.1.JNS131431.

Address correspondence to: Russell R. Lonser, M.D., Department of Neurological Surgery, Ohio State University Wexner Medical Center, 410 West 10th Ave., Doan Hall N1047, Columbus, OH 43210. email: russell.lonser@osumc.edu. 